\title{
Reactivity of lithium and platinum at elevated densities
}

\author{
Jack Binns, ${ }^{1}$ Miriam Marqués, ${ }^{2}$ Philip Dalladay-Simpson, ${ }^{1}$ Robin Turnbull, ${ }^{2}$ Mungo Frost, ${ }^{2, *}$ \\ Eugene Gregoryanz, ${ }^{2,1, \dagger}$ and Ross T. Howie ${ }^{1, \ddagger}$ \\ ${ }^{1}$ Center for High Pressure Science and Technology Advanced Research (HPSTAR), Shanghai 201203, People's Republic of China \\ ${ }^{2}$ Centre for Science at Extreme Conditions and The School of Physics and Astronomy, The University of Edinburgh, Peter Guthrie Tait Road, \\ Edinburgh EH9 3FD, United Kingdom
}

(Received 28 February 2019; published 10 June 2019)

\begin{abstract}
Through a series of x-ray diffraction experiments, combined with first-principles calculations, we show that the nobility of platinum can be overcome, reacting with lithium under compression at room temperature. Pressures as low as $2.3 \mathrm{GPa}$ lead to the synthesis of $\mathrm{Li}_{11} \mathrm{Pt}_{2}$, exhibiting the highest lithium content of any known intermetallic compound. Above $16 \mathrm{GPa}, \mathrm{Li}_{11} \mathrm{Pt}_{2}$ expels $\mathrm{Li}$ and transforms to $\mathrm{Li}_{2} \mathrm{Pt}$. This change is accompanied by a transformation in bonding characterized by the formation of one-dimensional Pt-Pt bonds with increasingly covalent character at higher densities.
\end{abstract}

DOI: 10.1103/PhysRevB.99.220101

Being the simplest and lightest metallic element, dense lithium $(\mathrm{Li})$ is of fundamental importance due to the strong influence of quantum effects on its behavior [1-8]. As other alkali metals, Li shows a pronounced minimum in the melting temperature with pressure, as low as $190 \mathrm{~K}$, leading to counterintuitive cold melting above $40 \mathrm{GPa}$ at room temperature $[7,9,10]$. When compressed at low temperatures, $\mathrm{Li}$ shows remarkable structural complexity, with at least five high-pressure configurations including low-symmetry semiconducting phases containing up to 88 atoms in the unit cell [6,7,11-13]. Platinum (Pt) is a highly unreactive transition metal with widespread industrial uses, such as in catalytic converters. Due to its stability and resistance to corrosion, it is widely used as a material for analytical standards such as pressure calibration, the standard hydrogen electrode in electrochemistry [14], and the now defunct International Prototype Kilogram and Meter which defined the base units of the metric system. Organometallic platinum compounds such as cisplatin and carboplatin play a role in cancer chemotherapy and owe their discovery to the use of an "inert" Pt electrode in a bacterial growth chamber $[15,16]$.

Compounds of the alkali and noble metals have been comprehensively studied since the early 20th century [17-19]. In these compounds, electron density is transferred from the electropositive alkali metal to the electronegative noble metal. Increasing differences in electronegativity lead to increasingly ionic behavior, reaching a logical conclusion in cesium auride $(\mathrm{CsAu})$ which is a yellowish transparent semiconductor with a band gap of $2.6 \mathrm{eV}$ [20]. After gold, platinum $(\mathrm{Pt})$ has the highest electron affinity of any metal [21]. Compounds of platinum with group 1 or 2 metals

*Current address: SLAC National Accelerator Laboratory, 2575 Sand Hill Road, Menlo Park, California 94025, USA.

†e.gregoryanz@ed.ac.uk

†ross.howie@hpstar.ac.cn exhibit significant charge transfer and can be considered as "platinides." While CsAu has a simple cubic structure, these compounds contain negatively charged Pt substructures such as $\mathrm{Pt}^{-2}$ anions, dimers ([Pt-Pt $]^{6-}$ ), and chains such as those found in $\mathrm{Li}_{2} \mathrm{Pt}$ [22-25]. Four $\mathrm{Li}-\mathrm{Pt}$ compounds are known at ambient pressures: $\mathrm{LiPt}_{7}, \mathrm{LiPt}_{2}, \mathrm{LiPt}, \mathrm{Li}_{2} \mathrm{Pt}$ all synthesized at high temperatures [25-27]. Unlike the $\mathrm{Au}-\mathrm{Li}$ and $\mathrm{Ag}-\mathrm{Li}$ systems, there are no known higher Li-content intermetallic compounds than the dilithide, $\mathrm{Li}_{2} \mathrm{Pt}[19,28]$. High-pressure reaction conditions have been an effective route to the formation of new compounds of the alkali metals [29,30] and could promote the formation of new high Li-content alloys for a range of applications such as energy storage [31,32].

Here, we show that these two archetypal metals, lithium and platinum, become highly reactive with the application of moderate pressures $(<3 \mathrm{GPa})$, resulting in the formation of an intermetallic compound $\mathrm{Li}_{11} \mathrm{Pt}_{2}$. This compound exhibits the highest Li content for any known intermetallic alloy which, combined with its low-pressure room-temperature synthesis conditions, suggests that these compounds may be overlooked as potential storage materials. With increasing pressure, $\mathrm{Li}_{11} \mathrm{Pt}_{2}$ transforms to the known compound $\mathrm{Li}_{2} \mathrm{Pt}$, driven by the formation of negatively charged one-dimensional PtPt chains as incompressible as pure Pt. The arrangement of these chains has been suggested to give rise to Dirac cones [33]. However, our electronic structure calculations show that these cones are "gapped out" at ambient conditions and increasing hydrostatic pressure exacerbates this breakdown of semimetallic behavior.

All experiments were carried out with excess Li concentrations (estimated 1\%-2\% Pt by volume). A detailed description of the sample preparation, experimental data collection, and theoretical procedures are given in the Supplemental Material (SM) [34]. Under these conditions pressures as low as $2.3 \mathrm{GPa}$ lead to a complete reaction of Pt. X-ray diffraction data collected at this pressure [Fig. 1(a)] indicate the formation of a compound crystallizing in cubic space group $I \overline{4} 3 \mathrm{~m}$, $a=9.2056(1) \AA$ A. Structure solution by charge flipping [35] 
(a)

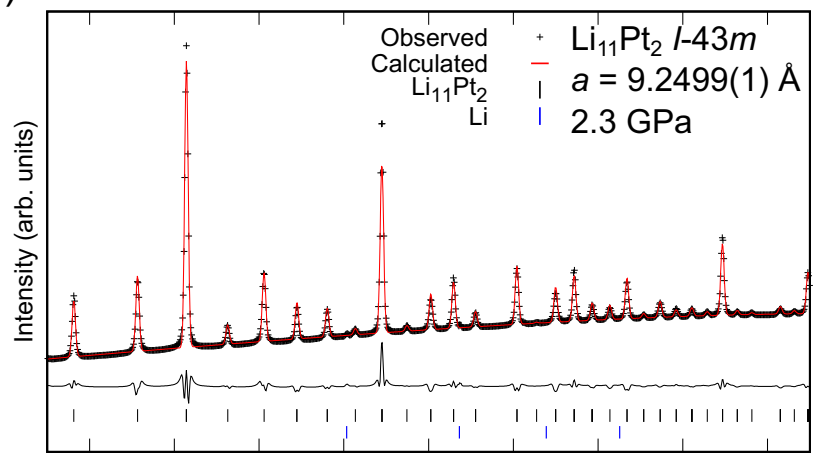

(b)

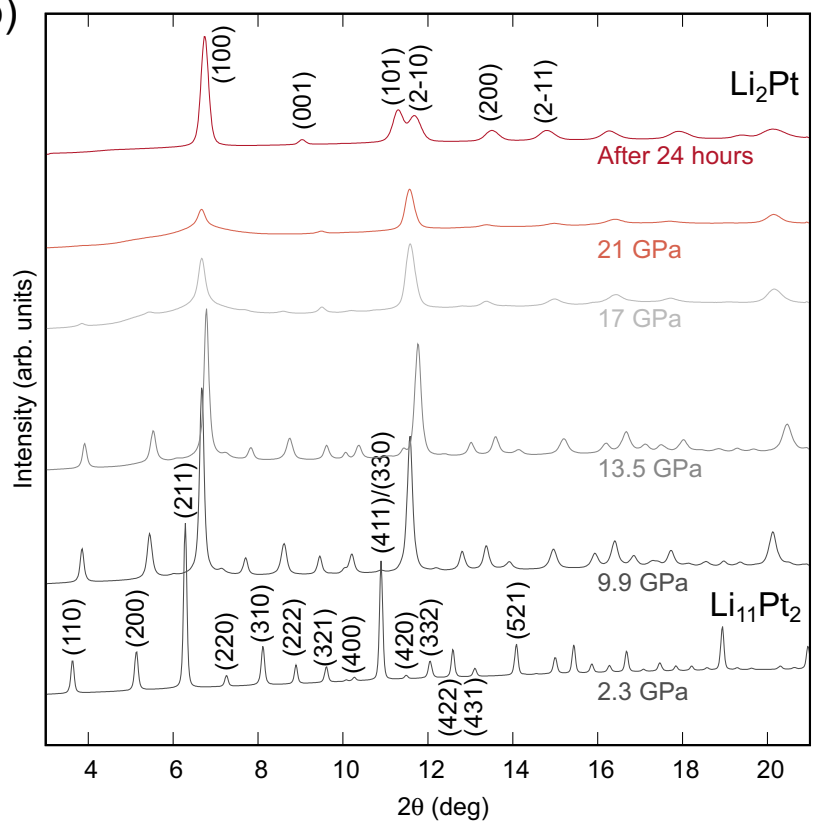

FIG. 1. High-pressure $x$-ray diffraction data on $\mathrm{Li}_{11} \mathrm{Pt}_{2}$. (a) $\mathrm{Ri}$ etveld profile refinement $(\lambda=0.4141 \AA)$ for $\mathrm{Li}_{11} \mathrm{Pt}_{2}\left(w R_{p}=3.84 \%\right.$, $R_{\text {expt }}=3.66 \%$ ): Experimental data shown with crosses, calculated profile shown in red, and the difference is shown as the lower black trace (raw x-ray diffraction image is shown in Fig. S1). (b) Diffraction patterns under increasing quasihydrostatic pressure. Peaks due to $\mathrm{Li}_{11} \mathrm{Pt}_{2}$ (bottom, black) broaden and rapidly lose intensity.

indicates eight $\mathrm{Pt}$ atoms per unit cell on the $8 c$ site. Three possible Li-atom sites were identified in Fourier difference maps giving a total of $44 \mathrm{Li}$ atoms in the unit cell. Density functional theory (DFT) calculations confirm the stoichiometry of this phase, $\mathrm{Li}_{11} \mathrm{Pt}_{2}$ (experimental and optimized atomic positions are given in Tables S1 and S2). Geometry optimization performed at increasing pressures show excellent agreement with the experimentally determined unit-cell length [Fig. 2(a)].

$\mathrm{Li}_{11} \mathrm{Pt}_{2}$ adopts a $\gamma$-brass-type structure which has been observed in a number of transition-metal alloys (e.g., $\mathrm{Zn}_{11} \mathrm{Ir}_{2}$, $\mathrm{Zn}_{11} \mathrm{Pt}_{2}$, etc. [36,37]) but has not been observed with an alkali metal. $\mathrm{Li}_{11} \mathrm{Pt}_{2}$ also displays the highest $\mathrm{Li}$ content of any Licontaining intermetallic [38-41]. The unit cell of $\mathrm{Li}_{11} \mathrm{Pt}_{2}$ contains two 26-atom clusters arrayed on a body-centered lattice. Each cluster is constructed from four edge- and face-sharing

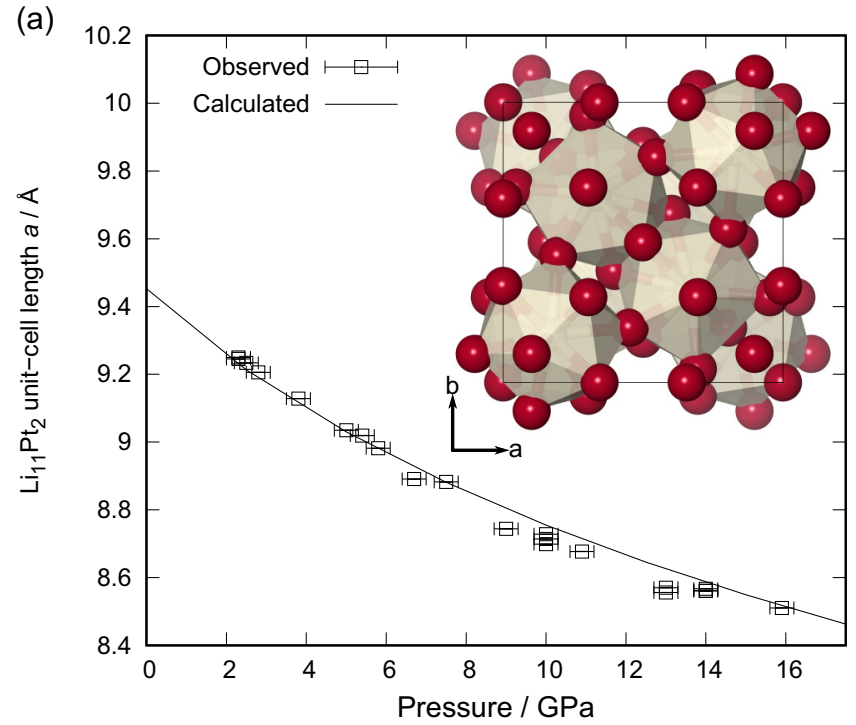

(b)

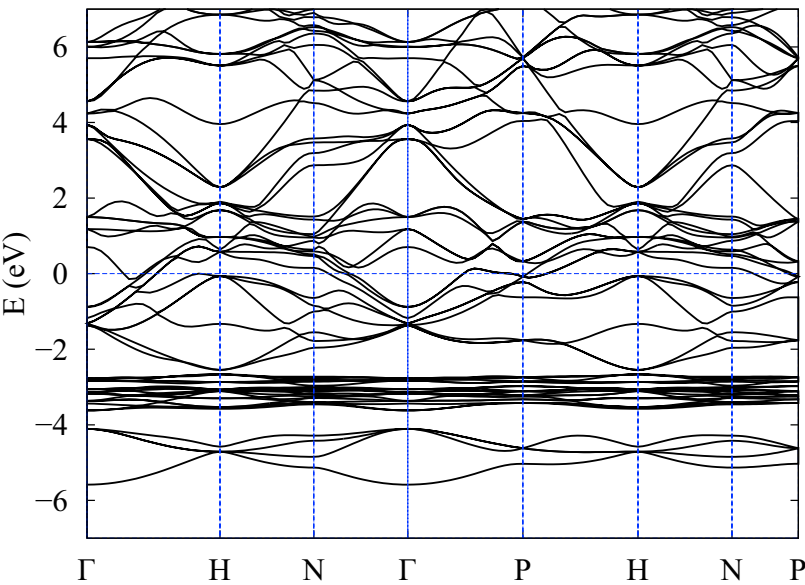

FIG. 2. Crystal and electronic structure of $\mathrm{Li}_{11} \mathrm{Pt}_{2}$. (a) Change in unit-cell lengths with pressure for $\mathrm{Li}_{11} \mathrm{Pt}_{2}$ : The inset shows the $\mathrm{Li}_{11} \mathrm{Pt}_{2}$ crystal structure, $\mathrm{Li}$ atoms are red, and $\mathrm{Pt}$ atoms beige. (b) Band structure of $\mathrm{Li}_{11} \mathrm{Pt}_{2}$ at $10 \mathrm{GPa}$.

irregular $\mathrm{PtLi}_{12}$ icosahedra [inset of Fig. 2(a)]. As a result of its extremely high $\mathrm{Li}$ content, $\mathrm{Li}_{11} \mathrm{Pt}_{2}$ is highly compressible and its unit-cell volume decreases by $20.9 \%\left(-163.7 \AA^{3}\right)$ between 2.8 and $15.9 \mathrm{GPa}$. Volume versus pressure data were fitted with a third-order Birch-Murnaghan equation of state giving a bulk modulus of 24(4) GPa, only twice that of pure Li [10.43(24) GPa] [42], suggesting the mechanical properties are dominated by $\mathrm{Li}-\mathrm{Li}$ interactions.

The electronic band structure of $\mathrm{Li}_{11} \mathrm{Pt}_{2}$ is shown in Fig. 2(b) and can be split into three regions: -5.6 to $-4.1 \mathrm{eV}$ corresponds mainly to the $\mathrm{Pt} 6 s$ band; -3.6 to $-2.6 \mathrm{eV}$ corresponds to the $\mathrm{Pt} 5 d$ band; and above. This remaining region above $-2.6 \mathrm{eV}$ is contributed to by all the bands, although the density of states (DOS) at $E_{F}$ is dominated by contributions from the Pt $5 d$ and Li $2 p$ orbitals. Interestingly, this structure shows similarities to the band structures of $\mathrm{Zn}_{11} \mathrm{Ni}_{2}$ and $\mathrm{Zn}_{11} \mathrm{Pd}_{2}$. The stabilities of these phases have been explained by Fermi-surface Brillouin-zone interactions involving $\{330\}$ and $\{411\}$ lattice planes and, similarly in $\mathrm{Li}_{11} \mathrm{Pt}_{2}$, the $d$ states are well immersed below the Fermi level. 
The metallic character of $\mathrm{Li}_{11} \mathrm{Pt}_{2}$ is also manifested by a flatness index of $\approx 0.4$ for all studied pressures. See SM for a full Bader analysis of $\mathrm{Li}_{11} \mathrm{Pt}_{2}$.

Within its coordination polyhedra, each $\mathrm{Pt}$ atom is surrounded by valence electron localization function (ELF) attractors (pink spheres in Fig. S2). The integration of the electron density within their corresponding chemical basins plus that contained in the electronic core leads to the $\mathrm{Pt}$ charge values of Table S4. Most importantly, the valence ELF attractors with the highest ELF value (0.615 at $0 \mathrm{GPa}$ ) appear outside the Pt coordination polyhedra and are almost coincident with the non-nuclear maxima (NNM) of the electron density (blue spheres in Fig. S2). Moreover, the localization window connecting them is very low $(0.03$ at $0 \mathrm{GPa})$. This generates a three-dimensional network of channels $\left(\eta_{\text {mesh }}\right.$ in Table S4) with an almost constant ELF. These characteristics are reminiscent of prototypical metallic systems such as bcc Li.

At pressures above $11 \mathrm{GPa}$, significant broadening of the $\mathrm{Li}_{11} \mathrm{Pt}_{2}$ peaks occurs, coinciding with the growth of a broad peak of diffuse scattering centered on the (211) reflection and which increases in intensity with pressure [Fig. 1(b)]. By $21 \mathrm{GPa}$, the peaks corresponding to $\mathrm{Li}_{11} \mathrm{Pt}_{2}$ are replaced by those from a different high-pressure phase. From this pressure, samples were decompressed to approximately $10 \mathrm{GPa}$ to avoid possible damage to the diamond anvils and left at room temperature for $24 \mathrm{~h}$. The sample was then compressed or decompressed to discern the stability range of this compound. X-ray diffraction surveys indicated some regions to be composed entirely of $\mathrm{Li}_{2} \mathrm{Pt}$, showing well-defined peak shapes and no diffuse scattering. All peaks from this highpressure phase are indexed to a hexagonal cell $a=3.8573(5)$, $c=2.6034(6) \AA$ (at $21 \mathrm{GPa}$ ), and subsequent Rietveld refinement confirms this phase to be $\mathrm{Li}_{2} \mathrm{Pt}$ crystallizing in space group $\mathrm{P6} / \mathrm{mmm}\left(\mathrm{CaHg}_{2}\right.$ type), where $\mathrm{Li}$ atoms lie on the $2 d$ sites $\left(\frac{1}{3}, \frac{2}{3}, \frac{1}{2}\right)$ with $\mathrm{Pt}$ atoms lying on the $1 a$ site $(0,0,0)$ [Fig. 3(a)]. $\mathrm{Li}_{2} \mathrm{Pt}$ is known to exist at ambient pressures and is formed by the reaction of $\mathrm{LiH}$ and $\mathrm{Pt}$ at temperatures of $870 \mathrm{~K}$ [25], however, here pressure is used to drive its formation at room temperature. The structure shows no variable positional parameters, therefore all structural parameters (i.e., bond distances, angles etc.) are fully determined by the values of the lattice constants. The structure of $\mathrm{Li}_{2} \mathrm{Pt}$ is characterized by linear chains of Pt atoms along the $c$ axis which pass through hexagonal, graphenelike sheets of Li atoms [Fig. 3(b)]. Both phases are stable to $3 \mathrm{GPa}$, the lowest pressure reached on decompression.

$\mathrm{Li}_{2} \mathrm{Pt}$ exhibits highly anisotropic compressibility; between 3 and $22 \mathrm{GPa}$ the $a$ axis reduces by $0.3339(7) \AA[-8.0 \%$, $\left.K_{a}=167(14) \mathrm{GPa}\right]$ and by contrast the $c$ axis is relatively incompressible, reducing by only $0.0466(8) \AA\left[-1.8 \%, K_{c}=\right.$ 319 (14) GPa] over the same pressure range. This behavior can be easily understood by considering changes in Pt-Pt distances and their relative compressibilities, as measured by linear moduli-one-dimensional equivalents to bulk moduli-with pressures as shown in Fig. S3(a). Pt-Pt distances between chains in $\mathrm{Li}_{2} \mathrm{Pt}$ are highly compressible, reducing by $8 \%$ up to $22 \mathrm{GPa}$, with a linear modulus of $47(9) \mathrm{GPa}$ which is comparable to those in $\mathrm{Li}_{11} \mathrm{Pt}_{2}$ [72(12) GPa]. By contrast, PtPt distances along $c$ are shorter than those found in elemental

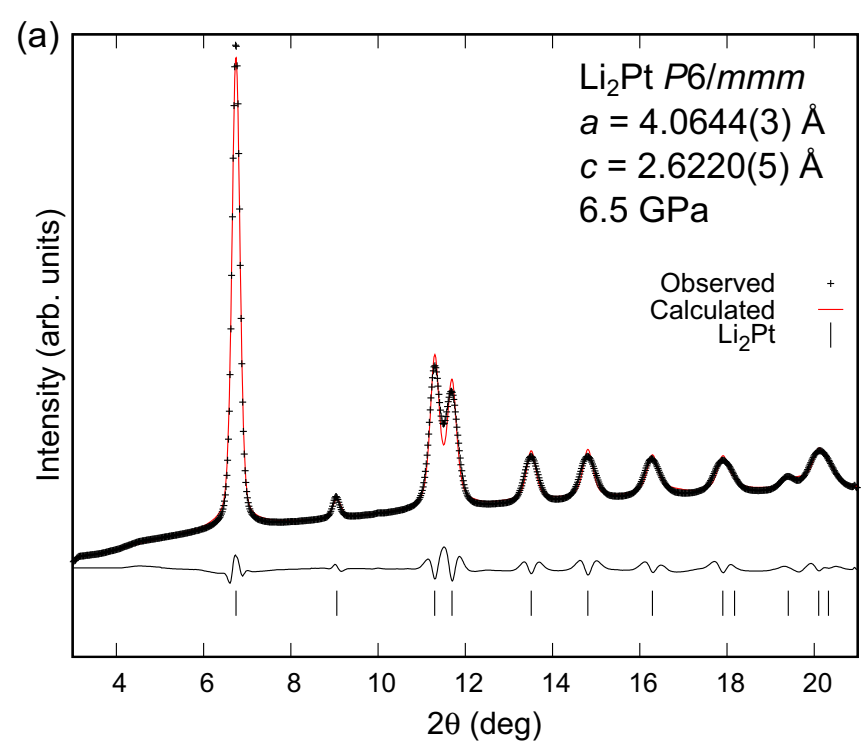

(b)



FIG. 3. High-pressure x-ray diffraction and crystal structure of $\mathrm{Li}_{2} \mathrm{Pt}$. (a) Rietveld profile refinement $(\lambda=0.4141 \AA$, raw x-ray diffraction image is shown in Fig. S1) for $\mathrm{Li}_{2} \mathrm{Pt}\left(w R_{p}=5.77 \%\right.$, $R_{\text {expt }}=3.35 \%$ ). (b) $2 \times 2$ supercell of $\mathrm{Li}_{2} \mathrm{Pt}$ : Chains of Pt atoms lie along the $c$ axis, each coordinated by $12 \mathrm{Li}$ atoms. Li atoms from graphenelike hexagonal sheets in the $a b$ plane.

Pt and are consequentially highly incompressible, reducing by just $2 \%$ over the same pressure range, with a linear modulus of $816(141) \mathrm{GPa}$ which is comparable to $795 \mathrm{GPa}$ for pure $\mathrm{Pt}$ [43].

$\mathrm{Li}_{2} \mathrm{Pt}$ has been proposed as a three-dimensional (3D) Dirac semimetal with a Dirac point at $K$ in analogy with graphene due to the linear chains of Pt anions ordered hexagonally in 2D [33]. Figures 4(a) and 4(b) show the band structures at 0 and $80 \mathrm{GPa}$ with spin-orbit coupling included. Although a Dirac point at $K$ appears at $0 \mathrm{GPa}$ when spin-orbit coupling is not considered, both pressure and spin-orbit coupling gap it out. The inclusion of spin-orbit coupling also leads to greater dispersion of the valence bands, particularly the lowest one along the reciprocal lines parallel to $c^{*}(\Gamma-A, H-K, L-M)$, associated with a strong interaction of the Pt atoms along the $c$ axis [Fig. 4(c)]. A partial ionic character is manifested by the flatness index as low as $\approx 0.13$, typical of ionic solids. See SI for a full Bader analysis of $\mathrm{Li}_{2} \mathrm{Pt}$.

The ELF topological analysis shows a hexagonal distribution of the ELF valence maxima around and between the 

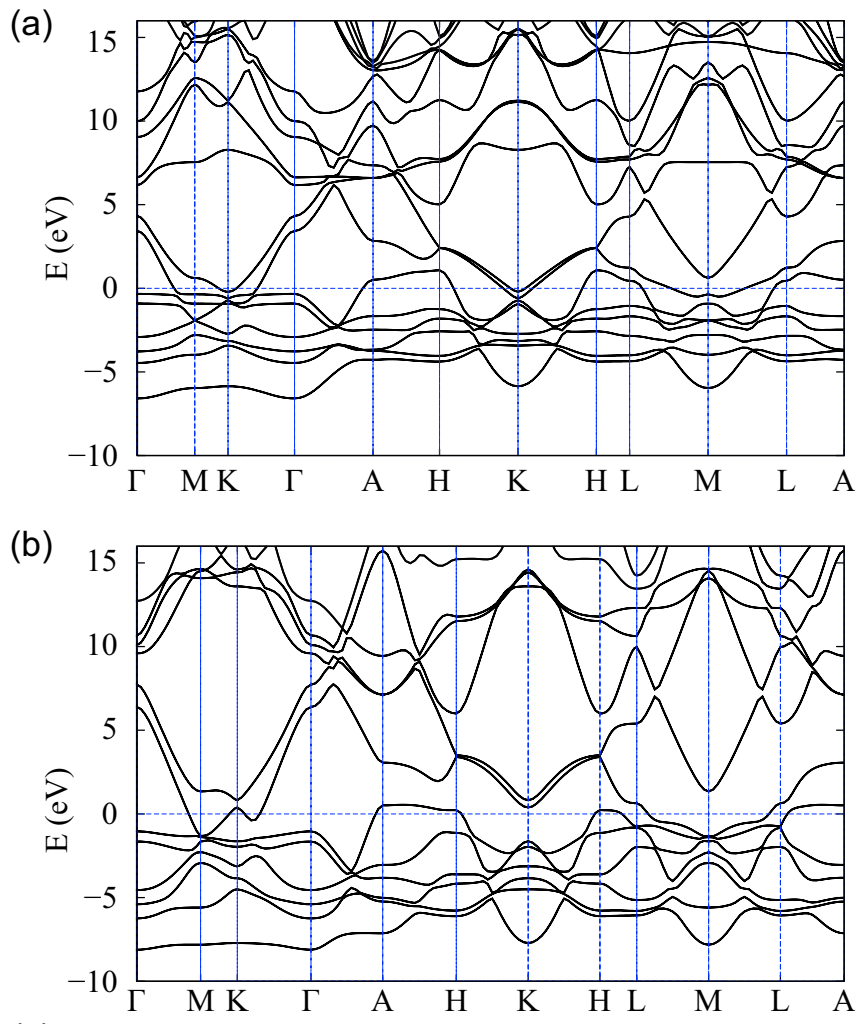

(c)

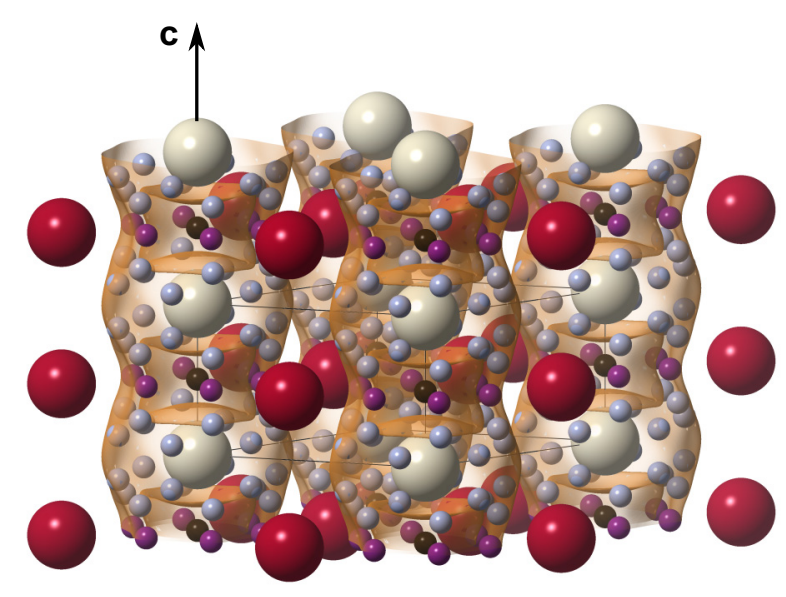

FIG. 4. Electronic structure of $\mathrm{Li}_{2} \mathrm{Pt}$ at (a) 0 and (b) $80 \mathrm{GPa}$. Pt $5 s, 5 p, 6 s, 5 d$ and Li $1 s, 2 s$ electrons are treated as valence. Spin-orbit coupling is included. (c) $\mathrm{ELF}=0.34$ isosurface for $\mathrm{Li}_{2} \mathrm{Pt}$ at $0 \mathrm{GPa}$. Valence ELF maxima are represented by blue balls. The valence ELF maxima located at $6 k$ and $1 b$ sites are indicated by purple and black balls, respectively.

Pt atoms [Fig. 4(c)]. This distribution generates channels of low ELF with a hexagonal prism shape along the Pt-Pt chains on the $c$ axis. We analyze the ELF value and integrated electron density of the valence ELF maxima that appear at the middle of the shortest Pt-Pt distance on the $c$ axis. Apart from the middle point ( $1 b$ site, black spheres), we have also considered the hexagon of ELF maxima ( $6 k$ sites, purple spheres) surrounding this $1 b$ site and conjugated to the $\mathrm{Li}_{6}$ hexagon coordinated to the $\mathrm{Pt}$ atoms at that $z$ coordinate. With increasing pressure these maxima show increased ELF value and electronic charge up to $40 \mathrm{GPa}$. A further detailed discussion of the ELF analysis is given in SM.

Regarding the implications of these results, conductivity measurements using $\mathrm{Pt}$ as the electrodes have been used to demonstrate wide ranging phenomena of $\mathrm{Li}$ at high pressure. These observations include superconductivity, high-pressure melting, and the influence of isotopic effects on $T_{c}[2,8,44,45]$. This work has shown that Li-Pt compounds are readily formed in Diamond-anvil cells (DACs) at $300 \mathrm{~K}$, and undergo structural transitions at modest pressures. It is highly likely that electronic measurements of $\mathrm{Li}$ are compromised at high pressure through sample contamination by the $\mathrm{Pt}$ (or indeed any noble metal) electrodes. Indeed, the possible contamination of $\mathrm{Li}$ at the contact point with $\mathrm{Pt}$ electrodes was postulated in early superconductivity measurements [2]. Simultaneous secondary diagnostics such as x-ray diffraction can be applied in order to definitively rule out pressure-induced chemical reactions [45], although measurements should be made sufficiently close to the electrode-sample interface to detect any reaction. Our observations may help explain the significant discrepancies that exist in the literature regarding the $\mathrm{Li}$ melting curve $[7,44]$ and the reportedly large isotopic effect on $T_{c}$ between ${ }^{6} \mathrm{Li}$ and ${ }^{7} \mathrm{Li}$ [8]. It should be noted that a subsequent theoretical study has been unable to reproduce the reported effect [46].

Our study has uncovered an alternative route to the synthesis of intermetallic compounds by compressing to moderate pressures at room temperature. At low pressures, we observe the formation of a compound $\mathrm{Li}_{11} \mathrm{Pt}_{2}$ which has the highest Li content for any known intermetallic compound. The low pressures required for synthesis suggest that this material could be used to store extensive quantities of $\mathrm{Li}$ for energy storage applications. Under further compression $\mathrm{Li}_{11} \mathrm{Pt}_{2}$ transforms to $\mathrm{Li}_{2} \mathrm{Pt}$, characterized by a chain of $\mathrm{Pt}$ atoms bonding in a quasi-one-dimensional manner resulting in highly anisotropic mechanical properties. Furthermore, our observation of strong pressure-driven reactivity between $\mathrm{Li}$ and $\mathrm{Pt}$ raises questions for a number of experiments measuring the electrical properties of $\mathrm{Li}$ at pressure using $\mathrm{Pt}$ electrodes $[2,8,44,45]$. In the absence of diagnostic x-ray diffraction confirming the purity of the Li samples studied, the formation of Li-Pt compounds in these experiments cannot be ruled out and it remains an open question what impact this will have on our current understanding of this fundamentally important system at high densities.

Parts of this research were carried out at P02.2 at DESY, a member of the Helmholtz Association (HGF). We would like to thank H.-P. Liermann and K. Glazyrin for assistance. Parts of this work was performed under Proposal No. 2017A1062 at SPring-8, and we thank S. Imada and N. Hirao for their assistance during experiments. Funding has been provided by the respective Chinese "1000 Talents Award" grants of both P.D.S. and R.T.H. M.M. acknowledges support from the ERC grant "Hecate" and computational resources provided by the UKCP consortium under the EPSRC grant (EP/P022561/1). R.T. acknowledges studentship funding from EPSRC Grants No. EP/L504956/1 and No. EP/M506515/1. 
[1] J. B. Neaton and N. W. Ashcroft, Nature (London) 400, 141 (1999).

[2] K. Shimizu, H. Ishikawa, D. Takao, T. Yagi, and K. Amaya, Nature (London) 419, 597 (2002).

[3] I. Tamblyn, J. Y. Raty, and S. A. Bonev, Phys. Rev. Lett. 101, 075703 (2008).

[4] S. F. Elatresh, W. Cai, N. W. Ashcroft, R. Hoffmann, S. Deemyad, and S. A. Bonev, Proc. Natl. Acad. Sci. USA 114, 5389 (2017).

[5] G. J. Ackland, M. Dunuwille, M. Martinez-Canales, I. Loa, R. Zhang, S. V. Sinogeikin, W. Cai, and S. Deemyad, Science 356, 1254 (2017).

[6] T. Matsuoka and K. Shimizu, Nature (London) 457, 186 (2009).

[7] C. L. Guillaume, E. Gregoryanz, O. Degtyareva, M. I. McMahon, M. Hanfland, S. Evans, M. Guthrie, S. V. Sinogeikin, and H.-K. Mao, Nat. Phys. 7, 211 (2011).

[8] A. M. Schaeffer, S. R. Temple, J. K. Bishop, and S. Deemyad, Proc. Natl. Acad. Sci. USA 112, 60 (2015).

[9] E. Gregoryanz, O. Degtyareva, M. Somayazulu, R. J. Hemley, and H. K. Mao, Phys. Rev. Lett. 94, 185502 (2005).

[10] E. Gregoryanz, L. F. Lundegaard, M. I. McMahon, C. Guillaume, R. J. Nelmes, and M. Mezouar, Science 320, 1054 (2008).

[11] B. Olinger and W. Shaner, Science 219, 1071 (1983).

[12] M. Marqués, M. I. McMahon, E. Gregoryanz, M. Hanfland, C. L. Guillaume, C. J. Pickard, G. J. Ackland, and R. J. Nelmes, Phys. Rev. Lett. 106, 095502 (2011).

[13] M. Hanfland, K. Syassen, N. E. Christensen, and D. L. Novikov, Nature (London) 408, 174 (2000).

[14] A. D. McNaught and A. Wilkinson, IUPAC Compendium of Chemical Terminology (Blackwell, Oxford, U.K., 1997).

[15] B. Rosenburg, L. Van Camp, and T. Krigas, Nature (London) 205, 698 (1965).

[16] L. Kelland, Nat. Rev. Cancer 7, 573 (2007).

[17] Thermal analysis of the system lithium-copper, S. Pastorello, Gazz. Chim. Ital. 60, 988 (1930).

[18] W. E. Spicer, A. H. Sommer, and J. G. White, Phys. Rev. 115, 57 (1959).

[19] G. Keinast, J. Verma, and W. Klemm, Z. Anorg. Allg. Chem. 310, 143 (1961).

[20] T. L. Liu, Phys. Rev. B 12, 3008 (1975).

[21] T. Andersen, H. Haugen, and H. Hotop, J. Phys. Chem. Ref. Data 28, 1511 (1999).

[22] A. Karpov, J. Nuss, U. Wedig, and M. Jansen, Angew. Chem., Int. Ed. 42, 4818 (2003).
[23] C. Lee, M.-H. Whangbo, and J. Köhler, J. Comput. Chem. 29, 2154 (2008).

[24] D. Kaczorowski, P. Rogl, and K. Hiebl, Phys. Rev. B 54, 9891 (1996).

[25] W. Bronger, B. Nacken, and K. Ploog, J. Less-Common Met. 43, 143 (1975).

[26] C. P. Nash, F. M. Boyden, and L. D. Whittig, J. Am. Chem. Soc. 82, 6203 (1960).

[27] W. Bronger, G. Klessen, and P. Müller, J. Less-Common Met. 109, L1 (1985).

[28] V. V. Pavlyuk, G. S. Dmytriv, I. I. Tarasiuk, I. V. Chumak, H. Pauly, and H. Ehrenberg, Solid State Sci. 12, 274 (2010).

[29] K. Takemura and H. Fujihisa, Phys. Rev. B 84, 014117 (2011).

[30] L. J. Parker, T. Atou, and J. V. Badding, Science 273, 95 (1996).

[31] J. Binns, P. Dalladay-Simpson, M. Wang, E. Gregoryanz, and R. T. Howie, J. Phys. Chem. Lett. 9, 3149 (2018).

[32] P. Dalladay-Simpson, J. Binns, M. Wang, M. Peña-Alvarez, E. J. Pace, E. Gregoryanz, X.-J. Chen, and R. Howie, J. Chem. Phys. 149, 024306 (2018).

[33] Q. D. Gibson, L. M. Schoop, L. Muechler, L. S. Xie, M. Hirschberger, N. P. Ong, R. Car, and R. J. Cava, Phys. Rev. B 91, 205128 (2015).

[34] See Supplemental Material at http://link.aps.org/supplemental/ 10.1103/PhysRevB.99.220101 for sample preparation, experimental data collection, and theoretical procedures.

[35] G. Oszlányi and A. Süto, Acta Crystallogr. A 60, 134 (2004).

[36] L. Arnberg and S. Westman, Acta Chem. Scand. 26, 513 (1972).

[37] B. Harbrecht, S. Thimmaiah, M. Armbrüster, C. Pietzonka, and S. Lee, Z. Anorg. Allg. Chem. 628, 2744 (2002).

[38] A. J. Bradley and J. Thewlis, Proc. R. Soc. London, Ser. A 112, 678 (1926).

[39] H. Perlitz, Z. Kristallogr. - Cryst. Mater. 86, 155 (1933).

[40] T. Noritake, M. Aoki, S.-i. Towata, T. Takeuchi, and U. Mizutani, Acta Crystallogr. B 63, 726 (2007).

[41] U. Mizutani, R. Asahi, H. Sato, T. Noritake, and T. Takeuchi, J. Phys.: Condens. Matter 20, 275228 (2008).

[42] M. Frost, A. L. Levitan, P. Sun, and S. Glenzer, J. Appl. Phys. 123, 065901 (2018).

[43] N. Holmes, J. Moriarty, G. Gathers, and W. Nellis, J. Appl. Phys. 66, 2962 (1989).

[44] A. M. J. Schaeffer, W. B. Talmadge, S. R. Temple, and S. Deemyad, Phys. Rev. Lett. 109, 185702 (2012).

[45] S. Deemyad and R. Zhang, Physica C 548, 68 (2018).

[46] M. Borinaga, U. Aseginolaza, I. Errea, M. Calandra, F. Mauri, and A. Bergara, Phys. Rev. B 96, 184505 (2017). 